\title{
Optimisation of the Alignment Sensitivity and Energy Stability of the NIF Regenerative Amplifier
}

\author{
N. W. Hopps, R. B. Wilcox, M. R. Hermann, \\ M. D. Martinez, E. H. Padilla, and J. K. Crane
}

This paper was prepared for and presented at the Third Annual International Conference on Solid State Lasers for Application (SSLA) to Inertial Confinement Fusion (ICF)

Monterey, CA

June 7-12, 1998

June 24, 1998

This is a preprint of a paper intended for publication in a journal or proceedings. Since changes may be made before publication, this preprint is made available with the understanding that it will not be cited or reproduced without the permission of the author. 


\section{DISCLAIMER}

This document was prepared as an account of work sponsored by an agency of the United States Government. Neither the United States Government nor the University of California nor any of their employees, makes any warranty, express or implied, or assumes any legal liability or responsibility for the accuracy, completeness, or usefulness of any information, apparatus, product, or process

disclosed, or represents that its use would not infringe privately owned rights. Reference herein to any specific commercial product, process, or service by trade name, trademark, manufacturer, or otherwise, does not necessarily constitute or imply its endorsement, recommendation, or favoring by the United States Government or the University of California. The views and opinions of authors expressed herein do not necessarily state or reflect those of the United States Government or the University of California, and shall not be used for advertising or product endorsement purposes. 
Optimisation of the alignment sensitivity and energy stability of the NIF regenerative amplifier cavity

\author{
N. W. Hopps ${ }^{2}$, R. B. Wilcox ${ }^{1}$, M. R. Hermann ${ }^{1}$, \\ M. D. Martinez ${ }^{1}$, E. H. Padilla ${ }^{1}$, J. K. Crane ${ }^{1}$. \\ ${ }^{1}$ Lawrence Livermore National Laboratory, 7000 East Avenue, Livermore, CA 94550. USA \\ ${ }^{2}$ Atomic Weapons Establishment, Aldermaston, Berkshire, RG7 4PR. UK
}

\begin{abstract}
The work to improve the energy stability of the regenerative amplifier ('regen') for the National Ignition Facility is described. This includes a fast feed-forward system, designed to regulate the output energy of the regen by monitoring how quickly a pulse builds up over many round trips. Shot-to-shot energy fluctuations of all elements prior to (and including) the regen may be compensated for in this way, at the expense of a loss of approximately 50\%. Also included is a detailed study into the alignment sensitivity of the regen cavity, with the goal of quantifying the effect of misalignment on the output energy. This is done by calculating the displacement of the eigenmode by augmenting the cavity $\mathrm{ABCD}$ matrix with the misalignment matrix elements, E, F. In this way, cavity misalignment issues due to thermal loading of the gain medium are investigated. Alternative cavity designs, which reduce the alignment sensitivity and therefore the energy drift over periods of continuous operation, are considered. Alterations to the amplifier head design are also considered.
\end{abstract}

\title{
1. INTRODUCTION
}

The regen is the first bulk gain component of the National Ignition Facility (NIF) ${ }^{1}$. It is supplied with temporally shaped pulses from the all-fibre-based Master Oscillator Room (MOR), via a polarising distribution fibre. This MOR signal (referred to hereafter as the 'seed') is injected into a linear cavity, with a round trip time of $30 \mathrm{~ns}$. The seed is mode-matched with the eigenmode of the cavity. The optical pulse is polarisation-switched into the cavity and is amplified on each round trip. The pulse remains inside the regen cavity for $\sim 27$ round trips before it is switched out. The amplification is supplied by an endpumped LG760 Nd:glass rod. The pump source is a $\sim 4 \mathrm{~kW}$ diode array, which produces $350 \mu$ s pulses at a repetition rate of $1 \mathrm{~Hz}$. The pump is concentrated into the end of the rod by a lens duct and is contained within the gain medium by total internal reflection. The far end of the rod is HR coated for the pump wavelength $(\sim 800 \mathrm{~nm})$ so that the pump sees two passes of the rod. The single pass gain achieved is about 1.6. The seed energy is $\sim 1 \mathrm{~nJ}$ and the regen output is $\sim 20 \mathrm{~mJ}$.

The output of the regen is spatially shaped to give a square beam with the appropriate profile, and then injected into a $50 \mathrm{~mm}$ diameter, 4-pass amplifier, which increases the signal energy to 14 J. This pulse is then split into 4 beams, each of which is injected into a single main NIF beam-line at the transport spatial filter. The regen, beam-shaping and 4-pass amplifier form a self-contained unit known as the Pre-Amplifier Module (PAM), which is designed to be a line replaceable unit (LRU) in the NIF design. There are 48 PAMs in total.

The regen is the highest gain component of the NIF. Its total net gain exceeds $10^{7}$. This makes it a potential source of energy instability. Small changes in gain can become significant quickly when compounded over 27 round trips (54 gain passes). The efforts to overcome these instabilities constitute the topic of this paper. There are two types of regen instability that need to be addressed.

\subsection{Shot-to-shot energy instability}

The variation in PAM output energies on any given NIF shot must be $\pm 3 \%$ RMS, or less. This is known as the power balance budget. The 4-pass amplifier takes up $\pm 2 \%$ of this, which leaves a budget of $\pm 2.2 \%$ for the regen and the MOR seed. With no mechanisms to improve the output stability, the regen output varies by more than $\pm 5 \%$, on a shot-to-shot basis. Clearly, this needs to be improved. As will be shown later, this has been done using a fast-feed-forward system, which monitors the power build-up in the cavity, whilst the pulse is trapped inside, and then based on this information, modulates the output pulse to achieve stable performance.

\subsection{Energy drift}

When the regen is turned on from a cold start, the output energy undergoes a gradual but dramatic fall. This drop is as great as $70 \%$ of the initial value, and the regen does not reach equilibrium for more than one hour. This paper will demonstrate that 
this effect is due to warming of the pump diodes, causing a slight reduction in diode efficiency and also a red-shift of the pump wavelength, which moves the diode spectrum away from the peak of the glass absorption curve. A small shift in the eigenmode of the cavity is also observed, caused by thermally induced pointing errors in the rod, but this will be shown to have a negligible effect on both the regen energy and pointing. Active cooling of the diode array will be shown to solve the energy drift problem.

\section{FAST-FEED-FORWARD ENERGY STABILISATION}

The fast-feed-forward system employed, was based around a component known as an 'Auston switch' ${ }^{2}$. This is essentially a small wafer of silicon (dimensions $10 \mathrm{~mm} \times 7 \mathrm{~mm}$ ) which is used to monitor the power build-up in the regen through one of the cavity folding mirrors. This information is then passed to a Pockels cell and used to control the voltage across it, in order to regulate the transmitted energy. A schematic of the optical arrangement is shown in figure 1.

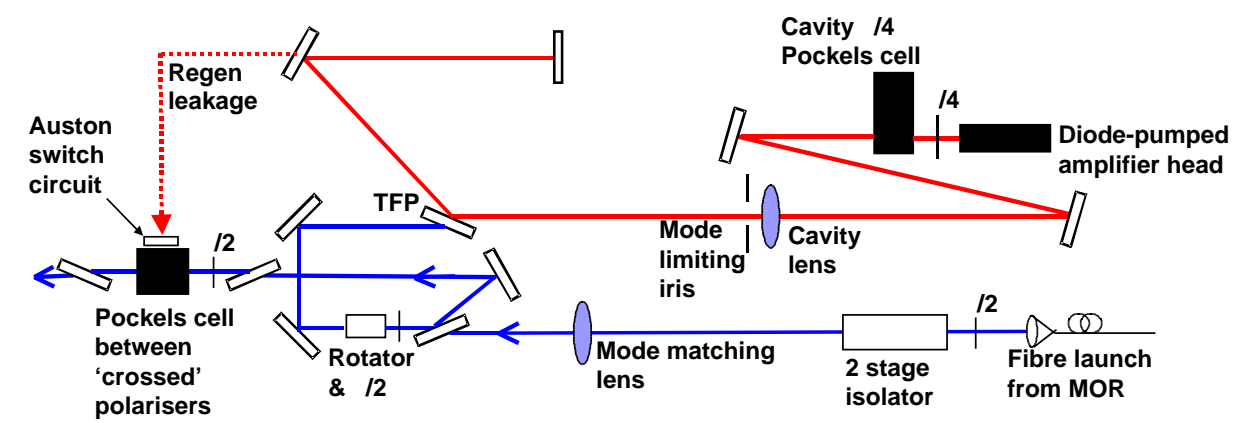

Figure 1: Optical arrangement showing regen cavity, input and output beams, and the Auston switch Pockels cell.

Photons that have leaked from the regen cavity, during the pulse build-up, are directed onto the Auston switch where they excite charge carriers from the valence to the conduction band of the semiconductor. This results in a drop in resistance that is dependent on the energy of the regen leakage. The switch integrates the photons it sees because the charge carriers have a long lifetime in the conduction band $(\sim 10 \mu \mathrm{s})$. If a fixed fraction of incident photons excite charge carriers, then the conductivity of the Auston switch will be proportional to the regen leakage energy i.e.

$$
Z_{A S}=\frac{1}{Z_{\text {dark }}}+a E_{\text {leak }}{ }^{-1}
$$

where $Z_{A S}$ is the Auston switch impedance, $Z_{\text {dark }}$ is the impedance with no illumination and $a$ is some constant which combines the transmission through the leaky mirror and the constant of proportionality between the switch conductivity and the illumination energy.

The switch is incorporated into a potential divider circuit, in series with some load resistance, $Z_{\text {load }}$. A dual-crystal Pockels cell is attached across the Auston switch. A $30 \mathrm{~ns} \mathrm{HV}$ pulse is supplied to the circuit, as shown.

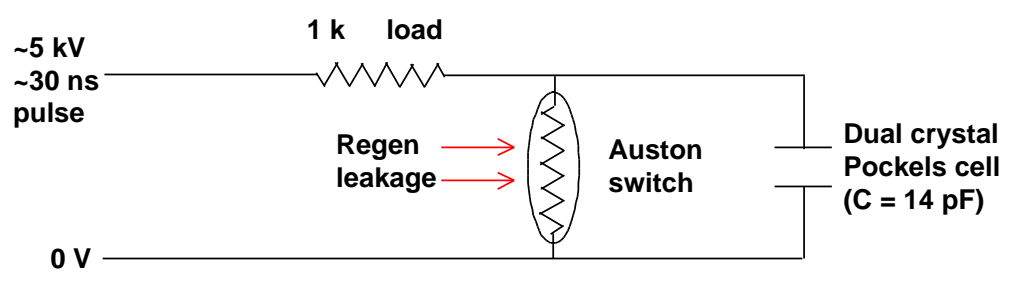

Figure 2: The Auston switch circuit.

The capacitance involved is small enough for the voltage across the Auston switch (and therefore the Pockels cell) to be represented as:

$$
V_{A S}=V_{\text {in }} \frac{Z_{A S}}{\left(Z_{A S}+Z_{\text {load }}\right)}
$$


where $V_{i n}$ is the input voltage. Note that since this is a double-sided Pockels cell, the half-wave voltage is only $\sim 4 \mathrm{kV}$. The Pockels cell is situated between crossed polarisers, so that the transmission through this system is:

$$
T=\sin ^{2} \frac{V_{A S}}{V_{\lambda / 2}} ? \frac{\pi}{2} \sqrt{ }
$$

Using the above three equations, the performance of the Auston switch can be accurately modelled, given realistic regen energetics data. Qualitatively, if the regen energy is higher than expected, this fact is detected by the Auston switch as a greater than expected amount of leakage. This results in lower than usual switch impedance and hence the Pockels cell voltage and transmission are reduced. Excesses in energy can therefore be balanced out by a reduction in Pockels cell transmission, if the system is set up correctly. This balance also works for lower than expected energies, of course. The results of an accurate model of the regen energetics are shown in figure 3 . This model takes into account saturation effects and the round-trip loss of the cavity. The growth of the signal, round trip by round trip,

begins exponentially because no significant stored energy has been extracted from the rod and its gain remains constant. At higher energies, the rod gain falls away. Eventually, the round trip gain cannot overcome the inherent round trip loss and the regen output falls. This point is known as the peak of saturation. Note the energy of the leakage is proportional to the area under the curve in figure 3. Beyond the peak of saturation, therefore, the leakage energy rises as the output energy falls.

The results of the Auston switch model, using equations 1, 2, 3, are shown in figure 4 . The data points represent transmitted energy measurements taken at different regen energies. The regen energy was varied by changing the amount of seed that was allowed into the regen cavity, using a half-wave plate. The operating region of the system is where the gradient of the curve is close to zero. Here, large changes in regen energy are translated into small changes in transmitted energy and the system acts to suppress regen instability. A good fit of the data is provided by the model, showing that there have been no unrealistic assumptions made.

Figure 5 illustrates the effectiveness of the feed-forward system under realistic operating conditions. The system parameters were set to the operating region and the regen was operated for several minutes with detectors measuring the regen output and transmitted energy through the Pockels cell. The standard deviation of the regen energy was $\pm 5.1 \%$, but the Auston switch system reduced this to $\pm 1.6 \%$ for the transmitted energy. This improvement in stability was purchased with a loss of about $50 \%$. The value of $\pm 1.6 \%$ is inside the regen and MOR budget of $\pm 2.2 \%$ (see above), with a comfortable margin.

It should be noted that the measurement error is negligible when compared to the scatter values shown here. Work will continue into reducing the value of the transmitted energy standard

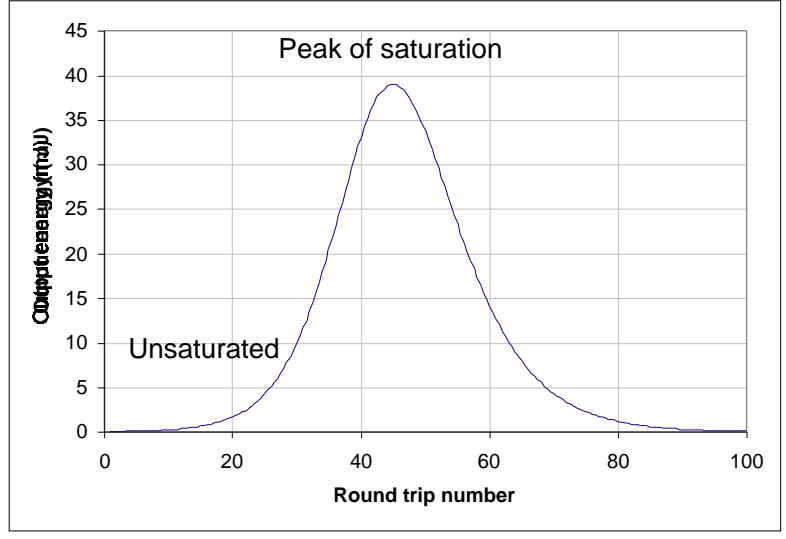

Figure 3: Regen output vs. round trip model results.

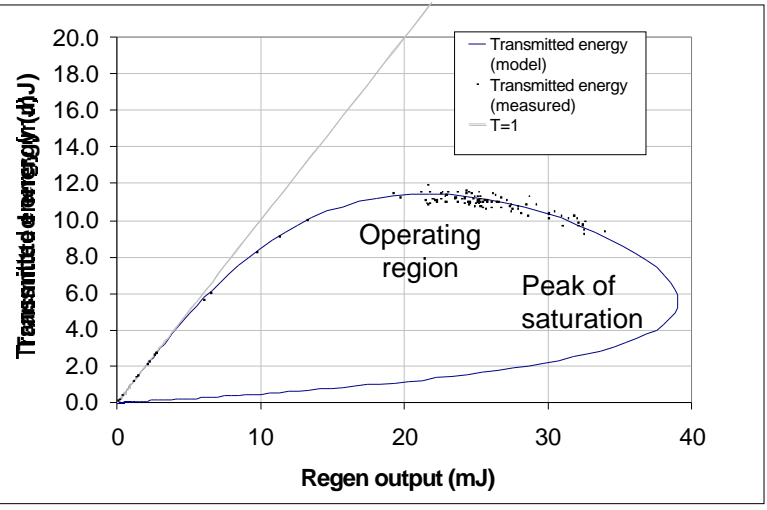

Figure 4: Feed-forward model data fit.

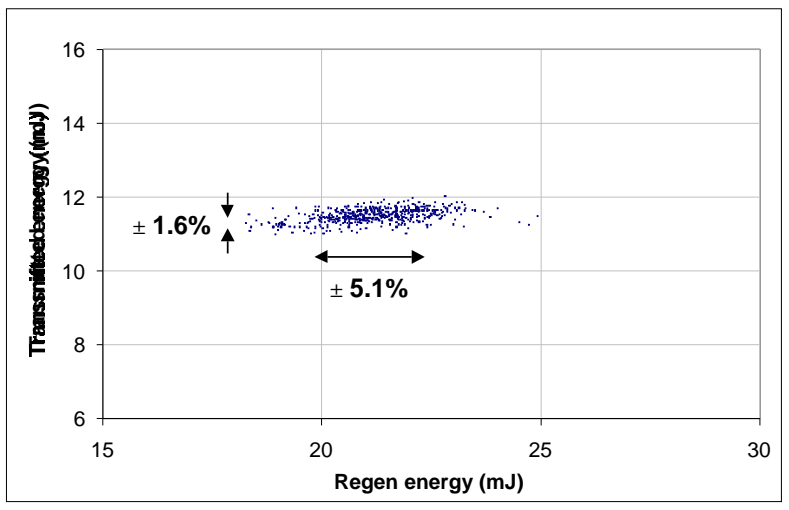

Figure 5: Feed-forward operation 
deviation further. It may be that if a larger silicon wafer was used, then the scatter would be lower since the resultant lower current density would cause less local heating of the switch, which also has an effect on the switch resistance.

\section{ENERGY DRIFT}

When the regen amplifier head is first turned on, there is a period during which it has not reached thermal equilibrium. There are two mechanisms by which thermal effects can influence the output energy of the regen. These are (i) a rising diode temperature causing lower efficiency and a red shift in the emission spectrum, away from the peak of glass absorption and (ii) thermally induced pointing errors in the rod, which can cause a displacement in the cavity eigenmode and, hence, greater round-trip loss. Both of these effects are investigated and their impact in the regen energy is assessed. Without any action to remedy the thermal issues in the head, the regen energy can drop by more than a factor of two, and take more than one hour to reach thermal equilibrium. In the context of day-to-day NIF operations, this delay would be problematical.

\subsection{Pump-diode thermal drift}

The pump light can be spectrally analysed by collecting some of the scattered pump light using a $50 \mu \mathrm{m}$ core fibre (with a collecting lens assembly), positioned near the interface between the lens duct and the rod. The other end of the fibre was positioned at the entrance plane of a $0.25 \mathrm{~m}$ grating spectrometer. A CCD camera detected the spectrometer output. The pump spectrum was roughly Gaussian in shape, FWHM of $\sim 4 \mathrm{~nm}$. The centre wavelength was seen to drift by as much as $3 \mathrm{~nm}$ before the array reached thermal equilibrium.

A temperature control mechanism was incorporated into the diode array assembly. A thermo-electric (TE) cooler was attached to the metal block onto which the array was mounted. This, in turn, was heat-sinked by a copper block, through which chilled water could be flowed. A thermistor monitored the temperature at a point close to the diode facets and fed this information back the TE cooler current source. A large range of operating temperatures could be attained using this thermal feedback circuit. A photodiode was used to monitor the power output of the array, so that any variation of this quantity with operating temperature could be ascertained. The experimental arrangement is illustrated in figure 6 .

Using this apparatus, the optimum pump wavelength could be ascertained. The diodes were operated at several different temperatures and, after waiting a few minutes to ensure the system was at equilibrium, the centre wavelength was measured in each case. The mean regen energy over 100 shots was calculated and plotted as a function of diode wavelength. This is shown in figure 7 . The peak of the absorption spectrum of the rod is at $801.5 \mathrm{~nm}$, as shown. The optimum pump wavelength, however, is some $2 \mathrm{~nm}$ shorter than this. This can be explained by the fact that the output power of the diodes, for a given current, increased at cooler temperatures. Over the range of data shown this increase was $\sim 10 \%$, measured with a photodiode. The curve in figure 7 is the output of a model which takes into account the overlap integral of the pump and absorption spectra, for different centre wavelengths, and also the variation of pump power with temperature. The model calculates the relative stored energy in the rod, in each case. From this, it infers the single pass gain and then the output energy of the regen, assuming unsaturated operation (this is a reasonable assumption, given the operating conditions of the regen at the time). The data

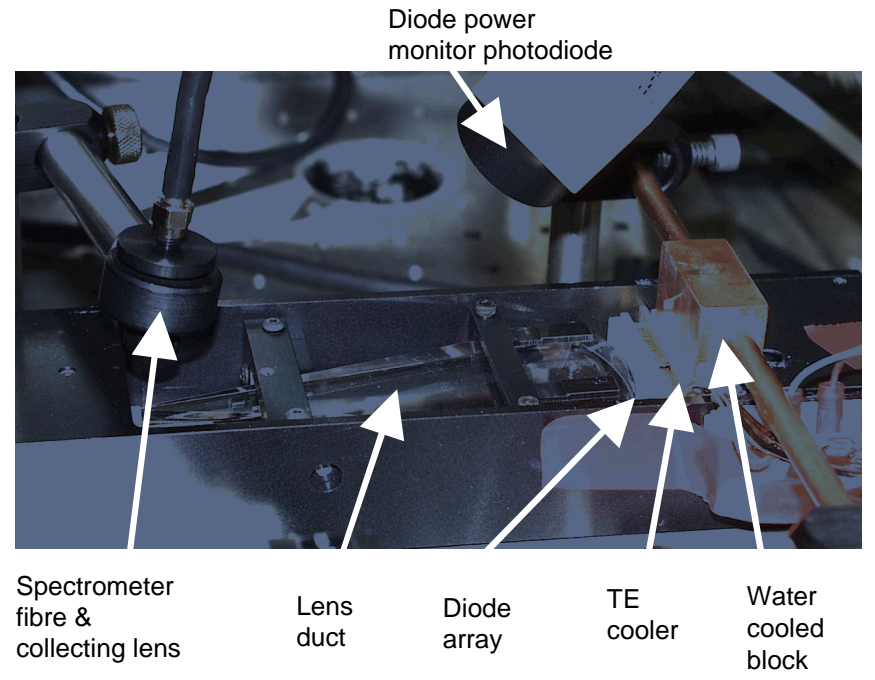

Figure 6: Pump diagnostics and temperature controller.

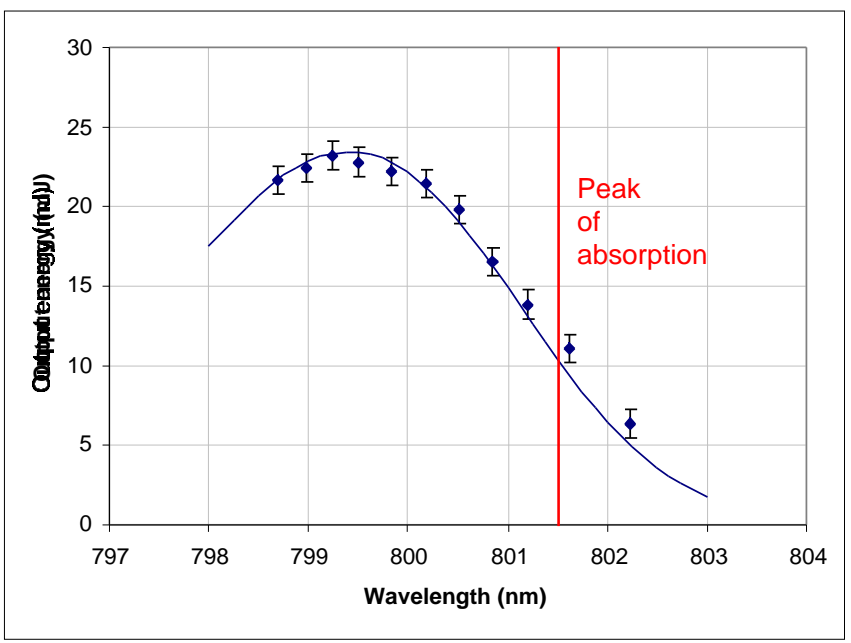

Figure 7: Regen output versus pump wavelength: data and model. 
and model show good agreement, suggesting that a good understanding of the processes involved has been achieved.

The warm-up cycles of the regen were compared without and with the cooling apparatus activated. The results are shown in figure 8 . When the temperature stabilisation was turned off, the regen energy dropped by roughly half its original value in about 45 minutes. The drift in pump wavelength mirrored the energy drop, with a rapid change initially, and then slowing as the system approached equilibrium. Activation of the temperature stabilisation reduced the warmup cycle to less than five minutes, which is a negligible period in the context of NIF operations. Holding the wavelength at near the optimum value prevented the output energy from falling, to any significant degree. Although the pump wavelength does show a small shift initially, this is quickly brought under control by the temperature stabilisation system and does not have a large effect on the output energy because the pump is close to the optimum wavelength, where regen output is insensitive to wavelength changes (see figure 7). Further drift of the output energy is negligible, which suggests that the diode temperature drift is the dominant factor influencing the output energy and that the displacement of the cavity eigenmode due to thermally induced pointing errors is not significant. Nevertheless, a study of this effect was carried out to confirm this hypothesis.

\subsection{Cavity eigenmode drift}

The displacement of the cavity eigenmode caused by a thermally induced pointing error in the rod can be calculated by determining the ABCD matrix of a round trip, and including the misalignment matrix elements $\mathrm{E}$ and $\mathrm{F}$. A round trip is defined as beginning immediately after the cavity lens and progressing to the end-mirror, back through the lens, onwards to the laser rod and returning to the make a second pass through the lens. The pointing error of the rod is represented by a simple mirror tilt at that position. This is illustrated in figure 9, along with the order in which the individual component ABCD matrices were applied. With the cavity represented in this way, the only component to which the misalignment elements E, F are applied is the second pass of the lens, which simplifies the analysis. This is true because E, F play no role for a tilted, flat mirror. The round trip ABDCEF matrix is given by the following equation.

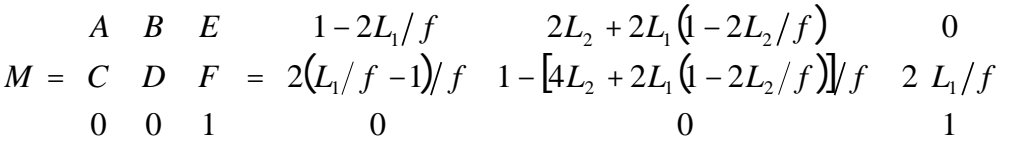

The angle $\theta$ represents the pointing error produced by the laser rod. Using this matrix, the displacement of the eigenmode at the cavity lens can be calculated. The mode-limiting iris in the cavity is situated very close to the lens. This is the point in the cavity where the mode sees the greatest loss. By calculating the offset of the mode at this point, the increase in loss at the iris due to the mode displacement can be ascertained. This is done by truncating a Gaussian profile with a hard, circular aperture (which represents the iris), with the two centres offset by some amount. Following Siegman ${ }^{3}$, the displacement of the eigenmode induced by a cavity with a round trip propagation matrix ABCDEF, is given by the following.

$$
\delta=\frac{(1-D) E+B F}{2-A-D}
$$


which, in this case, reduces to,

$$
\delta=\frac{L_{2}+L_{1}\left(1-2 L_{2} / f\right)}{L_{2}+L_{1}\left(1-L_{2} / f\right)} ? L_{1} \theta
$$

The angle, $\theta$, was estimated by reflecting a HeNe beam off the face of the rod and watching the displacement of this during the warm-up cycle of the head, using a CCD camera. A pointing displacement was observed, which settled down to a constant value (after about two hours) of $70 \mu \mathrm{rad}$. Equation 6 predicts a displacement of $60 \mu \mathrm{m}$ at the lens (also the iris). Simultaneously, the offset of the mode in the plane of the iris was measured directly, by imaging this plane onto another CCD camera. The mode displacement measured in this way agreed well with the predicted value, although the error of this measurement was large at $\pm 30 \%$.

A displacement of $60 \mu \mathrm{m}$ at the iris yields a negligible change in transmission through it. The calculated change is of the order of $0.05 \%$. When this value was inserted into the regen energetics model used to generate figure 3 , the change in output energy that resulted was roughly $1.5 \%$. This is less than the shot-to-shot variation and is therefore safe to ignore. A similar analysis can be applied to the question of pointing drift. Again the conclusion is that the pointing drift is roughly a factor of three below the shot-to-shot pointing stability, which has been measured as $\pm 6 \mu \mathrm{rad}$.

\section{CONCLUSIONS}

This paper has demonstrated the efficacy of a feed-forward-energy stabilisation technique, based on the Auston switch technology. Shot-to-shot energy fluctuations of $5.1 \%$ were reduced to $1.6 \%$ with this system. In broad terms therefore, the technique would be effective in this situation, provided that the regen fluctuations exceed the $1.6 \%$ level. At some point a judgement would have to be made on whether the loss in energy of about $50 \%$ is worth the increase in stability. In the context of the NIF requirements, however, some fluctuation technique is imperative in order to meet the power balance specifications.

The energy drift observed in the regen during its warm-up cycle has been shown to be overwhelmingly a consequence of diode warming. This affects both the operating wavelength and the power efficiency of the pump. An active cooling system has been shown to eliminate the warm-up period. Also, the optimum operating temperature has been established. This result is only valid for this particular array, however. With 48 PAMs in the NIF system, not all the arrays used may have the same wavelength for a given temperature. Again, this suggests that active cooling of the arrays is necessary if an optimised system is to be realised. Better performance would be achieved if future array purchases were specified to be longer in wavelength (at room temperature) than the peak of glass absorption. Advantage could then be taken of the improvements in efficiency and lifetime that result when the operating temperature of the diodes is reduced.

\section{ACKNOWLEDGEMENTS}

This work was done under the auspices of the U.S. Department of Energy by the Lawrence Livermore National Laboratory under Contract W-7405-ENG-48. The experiments were carried out during the secondment of N. W. Hopps from the Atomic Weapons Establishment in the UK.

\section{REFERENCES}

1. J. K. Crane, R. B. Wilcox, N. W. Hopps, D. Browning, M. D. Martinez, B. Moran, F. Penko, J. E. Rothenberg, M. Henesian, C. B. Dane, L. A. Hackel, "Integrated operations of the National Ignition Facility (NIF) optical pulse generation development system," $3^{\text {rd }}$ Annual conference on Solid State Lasers for application to Inertial Confinement Fusion, Monterey, CA, June 7-12, 1998.

2. P. Lefur and D. Auston, "A kilovolt picosecond optoelectronic switch and Pockels cell," Appl. Phys. Lett. 28(21), 21-23 (1976).

3. A. E. Siegman, Lasers, pp. 607-613, University Science Books, Mill Valley, 1986. 


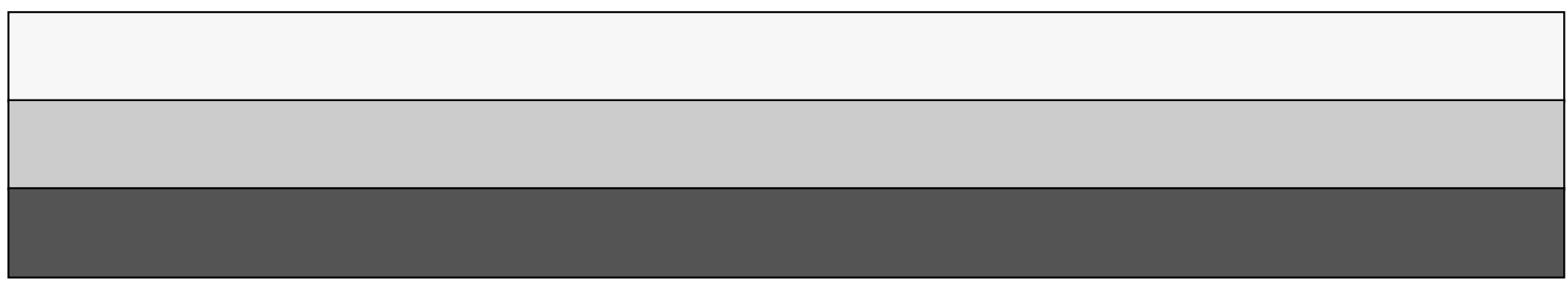

